\title{
Corticosterone rapidly increases thorns of CA3 neurons via synaptic/extranuclear glucocorticoid receptor in rat hippocampus
}

\author{
Miyuki Yoshiya ${ }^{1,2+}$, Yoshimasa Komatsuzaki ${ }^{1,3+}$, Yasushi Hojo ${ }^{1,2}$, Muneki Ikeda ${ }^{1}$, Hideo Mukai ${ }^{1,2}$, \\ Yusuke Hatanaka ${ }^{1,2}$, Gen Murakami ${ }^{1,2}$, Mitsuhiro Kawata ${ }^{4}$, Tetsuya Kimoto ${ }^{1}$ and Suguru Kawato ${ }^{1,2}$ * \\ ' Department of Biophysics and Life Sciences, Graduate School of Arts and Sciences, The University of Tokyo, Tokyo, Japan \\ ${ }^{2}$ Bioinformatics Project of Japan Science and Technology Agency, The University of Tokyo, Tokyo, Japan \\ ${ }^{3}$ Department of Physics, College of Science and Technology, Nihon University, Chiyoda, Tokyo, Japan \\ ${ }^{4}$ Department of Anatomy and Neurobiology, Kyoto Prefectural University of Medicine, Kamigyo, Kyoto, Japan
}

\section{Edited by:}

Yasuo Kawaguchi, National Institute

for Physiological Sciences, Japan

\section{Reviewed by:}

Yuji Ikegaya, The University of Tokyo, Japan

Janine Prange-Kiel, University of Texas Southwestern Medical Center, USA

\section{${ }^{*}$ Correspondence:}

Suguru Kawato, Department of Biophysics and Life Sciences,

Graduate School of Arts and Sciences,

The University of Tokyo, Komaba

3-8-1, Meguro, Tokyo 153, Japan

e-mail:kawato@bio.c.u-tokyo.ac.jp

${ }^{\dagger}$ Miyuki Yoshiya and Yoshimasa Komatsuzaki have contributed equally to this work.
Modulation of synapses under acute stress is attracting much attention. Exposure to acute stress induces corticosterone (CORT) secretion from the adrenal cortex, resulting in rapid increase of CORT levels in plasma and the hippocampus. We tried to test whether rapid CORT effects involve activation of essential kinases as non-genomic processes. We demonstrated rapid effects $(\sim 1 \mathrm{~h})$ of CORT on the density of thorns, by imaging Lucifer Yellow-injected neurons in adult male rat hippocampal slices. Thorns of thorny excrescences of CA3 hippocampal neurons are post-synaptic regions whose presynaptic partners are mossy fiber terminals. The application of CORT at 100, 500, and $1000 \mathrm{nM}$ induced a rapid increase in the density of thorns in the stratum lucidum of CA3 pyramidal neurons. Co-administration of RU486, an antagonist of glucocorticoid receptor (GR), abolished the effect of CORT. Blocking a single kinase, including MAPK, PKA, or PKC, suppressed CORTinduced enhancement of thorn-genesis. On the other hand, GSK-3 $\beta$ was not involved in the signaling of thorn-genesis. Blocking AMPA receptors suppressed the CORT effect. Expression of CA3 synaptic/extranuclear GR was demonstrated by immunogold electron microscopic analysis. From these results, stress levels of CORT (100-1000 nM) might drive the rapid thorn-genesis via synaptic/extranuclear GR and multiple kinase pathways, although a role of nuclear GRs cannot be completely excluded.

Keywords: corticosterone, hippocampus, kinase, thorn, stress, spine

\section{INTRODUCTION}

Functions and architectures of mammalian hippocampus are altered or modulated under the stressful conditions. At least in part, the influences of stress are elicited by corticosterone (CORT), produced in adrenal cortex in response to stress. The hippocampus, center for learning and memory, is particularly sensitive to CORT (Woolley et al., 1990b; Watanabe et al., 1992; Reagan and McEwen, 1997), because glucocorticoid receptors (GR) are abundantly expressed in the hippocampus (Morimoto et al., 1996). The chronic stress-induced increase in CORT slowly produces neuronal cell damage in the hippocampus. Rats exposed to restraint stress for 3 weeks have exhibited neuronal atrophy and decreases of dendritic branches similar to that seen in rats treated with a high dose of CORT for 3 weeks (Woolley et al., 1990b; Watanabe et al., 1992).

The CA3 is considered as a region where controls associative memory (Morris et al., 1982; McNaughton and Morris, 1987). In the stratum lucidum of the CA3, pyramidal neurons have huge and complex post-synaptic structures, named thorny excrescences. One thorny excrescence consists of multiple heads named thorns with one neck along a dendritic branch (Amaral and Dent, 1981; Chicurel and Harris, 1992). One mossy fiber terminal of dentate granule cells contacts multiple thorns of thorny excrescences of
CA3 neuron. Thorny excrescences may play essential roles in hippocampal function. Chronic restraint stress has induced retraction of thorny excrescences, which has subsequently been reversed after water maze training. On the other hand, water maze training alone has increased the volume of thorny excrescence as well as the number of thorns per thorny excrescence (Stewart et al., 2005). These slow steroid effects may be mediated by nuclear receptors. Upon binding of steroids to nuclear GR, GR forms dimer and bind to the glucocorticoid response element of genes, resulting in modulation of protein synthesis.

The neuronal response to acute stress (within a few hours) may be very different from that of chronic stress (Sorrells et al., 2009). CORT modulates rapidly (within $2 \mathrm{~h}$ ) the neuronal activity, which may occur independently of the regulation of the gene expression (Lupien and McEwen, 1997). Stress levels (500-1000 nM) of CORT have been demonstrated to rapidly suppress within $0.5 \mathrm{~h}$ the longterm potentiation (LTP) induced by primed burst stimulation (Diamond et al., 1992) or tetanic stimulation (Shibuya et al., 2003). A $0.5 \mathrm{~h}$ application of 1-10 $\mu \mathrm{M}$ CORT has rapidly suppressed the $\mathrm{N}$-methyl D-aspartate (NMDA)-induced $\mathrm{Ca}^{2+}$ elevation in the CA1 region of adult hippocampal slices (Sato et al., 2004). In our early study (Komatsuzaki et al., 2012), we demonstrated in CA1 that the application of CORT at $100-1000 \mathrm{nM}$ induces a rapid 
$(\sim 1 \mathrm{~h})$ increase in the density of spines of pyramidal neurons. Blocking kinases, including MAPK, PKA, and PKC, suppressed CORT-induced enhancement of spinogenesis. The receptor of this rapid CORT reaction is synaptic GR.

Compared to the CA1 region, little is known about the response of CA3 hippocampal thorns to the acute stress. We perform the investigations in order to examine the hypothesis that CORT may induce activation of synaptic/extranuclear GR, leading to activation of essential kinases, resulting in rapid remodeling of thorns in CA3 neurons.

\section{MATERIALS AND METHODS ANIMALS}

Male Wistar rats were purchased from Saitama Experimental Animal Supply (Japan). All animals were maintained under a $12 \mathrm{~h}$ light/12 $\mathrm{h}$ dark exposure and free access to food and water. The experimental procedure of this research was approved by the Committee for Animal Research of the University of Tokyo.

\section{CHEMICALS}

Corticosterone, actinomycin D, cyano-nitroquinoxaline-dione (CNQX), MK-801, PD98059, RU486, and Lucifer Yellow CH were purchased from Sigma (USA). Chelerythrine and glycogen synthase kinase-3 $\beta$ (GSK-3 $\beta$ ) inhibitor VIII (AR-A014418) were purchased from Calbiochem (Germany). H-89 was purchased from Biomol (USA).

\section{SLICE PREPARATION}

Twelve weeks male rats were deeply anesthetized and decapitated between 9:00 AM and 10:00 AM when plasma CORT levels are low. Immediately after decapitation, the brain was removed from the skull and placed in ice-cold oxygenated $\left(95 \% \mathrm{O}_{2}, 5 \% \mathrm{CO}_{2}\right)$ artificial cerebrospinal fluid (ACSF) containing (in $\mathrm{mM}$ ): 124 $\mathrm{NaCl}, 5 \mathrm{KCl}, 1.25 \mathrm{NaH}_{2} \mathrm{PO}_{4}, 2 \mathrm{MgSO}_{4}, 2 \mathrm{CaCl}_{2}, 22 \mathrm{NaHCO}_{3}$, and 10 D-glucose (all from Wako); $\mathrm{pH}$ was set at 7.4. Hippocampal slices, $400 \mu \mathrm{m}$ thick, were prepared with a vibratome (Dosaka, Japan). These slices were "freshly prepared" slices without ACSF incubation. Slices were then incubated for recovery in oxygenated ACSF for $2 \mathrm{~h}$ (slice recovery process) in order to obtain conventional "acute slices." These "acute" slices were then incubated at room temperature with CORT or other drugs such as kinase inhibitors. Immediately after drug exposure (for 0.5 , 1, or $2 \mathrm{~h}$ ), slices were prefixed with $4 \%$ paraformaldehyde at $4^{\circ} \mathrm{C}$ for $2-4 \mathrm{~h}$.

\section{CURRENT INJECTION OF LUCIFER YELLOW}

Thorn imaging and analysis with confocal microscopy was performed essentially as described previously (Komatsuzaki et al., 2005, 2012; Tsurugizawa et al., 2005; Mukai et al., 2007). Briefly, neurons within slices were visualized by an injection of Lucifer Yellow under a Nikon E600FN microscope (Japan) equipped with a C2400-79H infrared camera (Hamamatsu Photonics, Japan) and with a $40 \times$ water immersion lens (Nikon). Dye was injected with a glass electrode whose tip (tip diameter $<1 \mu \mathrm{m}$ ) was filled with 5\% Lucifer Yellow under a negative DC current of $10 \mathrm{nA}$ for $15 \mathrm{~min}$, using Axopatch 200B (Axon Instruments, USA). Approximately five neurons within a 100-200 $\mu \mathrm{m}$ depth from the surface of a slice were injected (Duan et al., 2002). After labeling, slices were fixed again with $4 \%$ paraformaldehyde at $4^{\circ} \mathrm{C}$ overnight.

\section{CONFOCAL LASER SCAN MICROSCOPY AND ANALYSIS}

The imaging was performed from sequential z-series scans with confocal laser scan microscope (LSM5; Carl Zeiss, Germany) at high zoom $(\times 3.0)$ with a $63 \times$ water immersion lens, NA 1.2. For Lucifer Yellow, the excitation and emission wavelengths were 488 and $515 \mathrm{~nm}$, respectively. Three-dimensional image was reconstructed from approximately 40 sequential z-series sections of every $0.45 \mu \mathrm{m}$. The applied zoom factor $(\times 3.0)$ yielded 23 pixels per $1 \mu \mathrm{m}$. The confocal lateral resolution was approximately $0.26 \mu \mathrm{m}$. Our resolution limits were regarded to be sufficient to allow the determination of the density of thorns. Confocal images were then deconvoluted using AutoDeblur software (AutoQuant, USA).

In each slice, two to three neurons with more than 100 thorns were analyzed, and at least 90 thorns were counted on each frame. In total, $N=12$ neurons and $n=1400-1800$ thorns were analyzed for each drug treatment. The density of thorns was analyzed with Spiso-3D developed by Bioinformatics Project of Kawato's group (Mukai et al., 2011; Komatsuzaki et al., 2012). Results obtained by Spiso-3D are similar to those by Neurolucida (MicroBrightField, USA) within assessment difference of $2 \%$, and Spiso-3D considerably reduces human errors and experimental labor of manual software (Mukai et al., 2011). The apical dendrite in the stratum lucidum has thorns. Such a dendrite (primary or secondary dendrite) is present within $100 \mu \mathrm{m}$ from the soma. The density of thorns was calculated from the number of thorns along the dendrite having a total length of 30-100 $\mu \mathrm{m}$. While counting the thorns in reconstructed images, the position and verification of thorns were aided by three-dimensional reconstructions and by observation of the images in consecutive single planes.

\section{POSTEMBEDDING IMMUNOGOLD METHOD FOR ELECTRON MICROSCOPY}

Immunoelectroscopic analysis was performed essentially as described elsewhere (Hojo et al., 2004; Mukai et al., 2007; Ooishi et al., 2012b). Rat hippocampus was frozen and sliced coronally. Freeze substitution and low-temperature embedding of the specimens was performed as described previously (Roberson et al., 1999). The samples were immersed in uranyl acetate in anhydrous methanol $\left(-90^{\circ} \mathrm{C}\right)$. The samples were infiltrated with Lowicryl HM20 resin (Electron Microscopy Sciences, USA) and polymerization was performed with ultraviolet light. Ultrathin sections were cut using a Reichert-Jung ultramicrotome. For immunolabeling, sections were incubated with primary antibody for GR (Morimoto et al., 1996; diluted to 1/3000) overnight, and incubated with secondary gold-tagged $(10 \mathrm{~nm})$ Fab fragment in Tris buffered saline (TBS). Sections were counterstained with $1 \%$ uranyl acetate, and viewed on a JEOL 1200EX electron microscope (Japan). Images were captured using a CCD camera (Advanced Microscopy Techniques, USA). The antibody is specific to GR in the hippocampus as shown with Western blot (Komatsuzaki et al., 2005; Ooishi et al., 2012b). 


\section{STATISTICAL ANALYSIS}

All the data are expressed as means \pm SEM. The significance of CORT or drug effect was examined using the Tukey-Kramer post hoc multiple comparisons test when one way ANOVA tests yielded $p<0.05$.

\section{RESULTS}

We investigated the effect of CORT on the modulation of the thorn density in the hippocampus CA3 stratum lucidum. Lucifer Yellow-injected neurons in hippocampal slices from 12-weekold male rats were imaged using confocal laser scan microscopy (Figure 1). Thorny excrescences were located on apical dendrites within $100 \mu \mathrm{m}$ from the soma, on which mossy fiber terminals attached.

\section{CORT INCREASED THE DENSITY OF THORNS IN CA3 STRATUM LUCIDUM}

Following a $1 \mathrm{~h}$ treatment with CORT, treated dendrites had significantly more thorns than control dendrites (i.e., $1 \mathrm{~h}$ incubation in ACSF without CORT). Time dependency was examined by treating slices for $0.5,1$, and $2 \mathrm{~h}$ with $1 \mu \mathrm{M}$ CORT. The enhancing effect on the total thorn density was approximately proportional to the incubation time, showing 2.7 $(0.5 \mathrm{~h}), 3.2 \mathrm{(}(\mathrm{h})$, and 3.2 thorns/ $\mu \mathrm{m}(2 \mathrm{~h})$ in CORT-treatments (Figure 2A). Dose dependency was also examined after a $1 \mathrm{~h}$ incubation (Figure 2B). In CORT-treatment group, the enhancing effect was significant at $1 \mu \mathrm{M}$ CORT (3.2 thorns/ $\mu \mathrm{m})$ compared with $10 \mathrm{nM}(2.4$ thorns $/ \mu \mathrm{m}), 30 \mathrm{nM}(2.9$ thorns $/ \mu \mathrm{m})$, $100 \mathrm{nM}$ (3.0 thorns/ $\mu \mathrm{m})$, and $500 \mathrm{nM}$ (3.3 thorns/ $\mu \mathrm{m})$ CORT. Because a $1 \mathrm{~h}$ treatment with $1 \mu \mathrm{M}$ CORT was most effective for thorn-genesis, these incubation time and concentration were used in the following investigations unless specified.

A $1 \mathrm{~h}$ treatment with $1 \mu \mathrm{M}$ CORT was used in the kinase inhibitor investigations unless specified, because $1 \mu \mathrm{M}$ CORT showed the strongest effects. Blocking of GR by $10 \mu \mathrm{M}$ RU486 completely abolished the enhancing effect by $1 \mu \mathrm{M}$ CORT on the thorn density (2.4 thorns/ $\mu \mathrm{m}$; Figure 3 ). It should be noted that rapid CORT effects (within $1 \mathrm{~h}$ ) did not induce neurodegeneration, as judged from no significant shrinking in dendrite length and atrophy of cell body (data not shown).

\section{EFFECT OF CORT WAS BLOCKED BY SEVERAL KINASE INHIBITORS}

Next we investigated kinase signaling pathways involved in the CORT-induced thorn-genesis using specific inhibitors for kinases (Figure 4), by examining the total thorn density. We focus on MAPK, PKA, and PKC, since these kinases often play an important role in synaptic plasticity. Blocking of Erk MAPK by application of $20 \mu \mathrm{M}$ PD98059 (PD; Dudley etal., 1995), abolished the CORT-induced increase in thorn density resulting in 2.6 thorns/ $\mu \mathrm{m}$. Application of $10 \mu \mathrm{M} \mathrm{H}-89$ (H89), a protein A kinase inhibitor (Chijiwa etal., 1990), prevented the effect by CORT. Application of $10 \mu \mathrm{M}$ chelerythrine (Chel), an inhibitor of all the PKC species (alpha, delta, and epsilon; Herbert et al., 1990), prevented the effect by CORT. Blocking of glycogen synthase kinase-3 $\beta$ (GSK-3 $\beta$ ) by $10 \mu \mathrm{M}$ Inhibitor VIII (I8; Bhat et al., 2003) did not alter CORT-induced thorngenesis. Effect of GSK-3 $\beta$, which is tau protein kinase, was investigated, since phosphorylation of tau protein (that stabilizes microtubules) is necessary for BDNF (brain-derived neurotrophic factor)-induced spinogenesis in the hippocampus (Chen et al., 2012).

Because the concentrations of inhibitors applied are recommended levels (Bhat et al., 2003; Alonso et al., 2004; Birnbaum et al., 2004; Hammond et al., 2008), the observed inhibitory effects are not artifacts due to excess amount of inhibitors. It should be noted that these kinase inhibitors alone did not significantly affect the thorn density within experimental error, indicating that the observed inhibitory effects are not due to simple blocker's non-specific suppressive effects (Figure 4B).

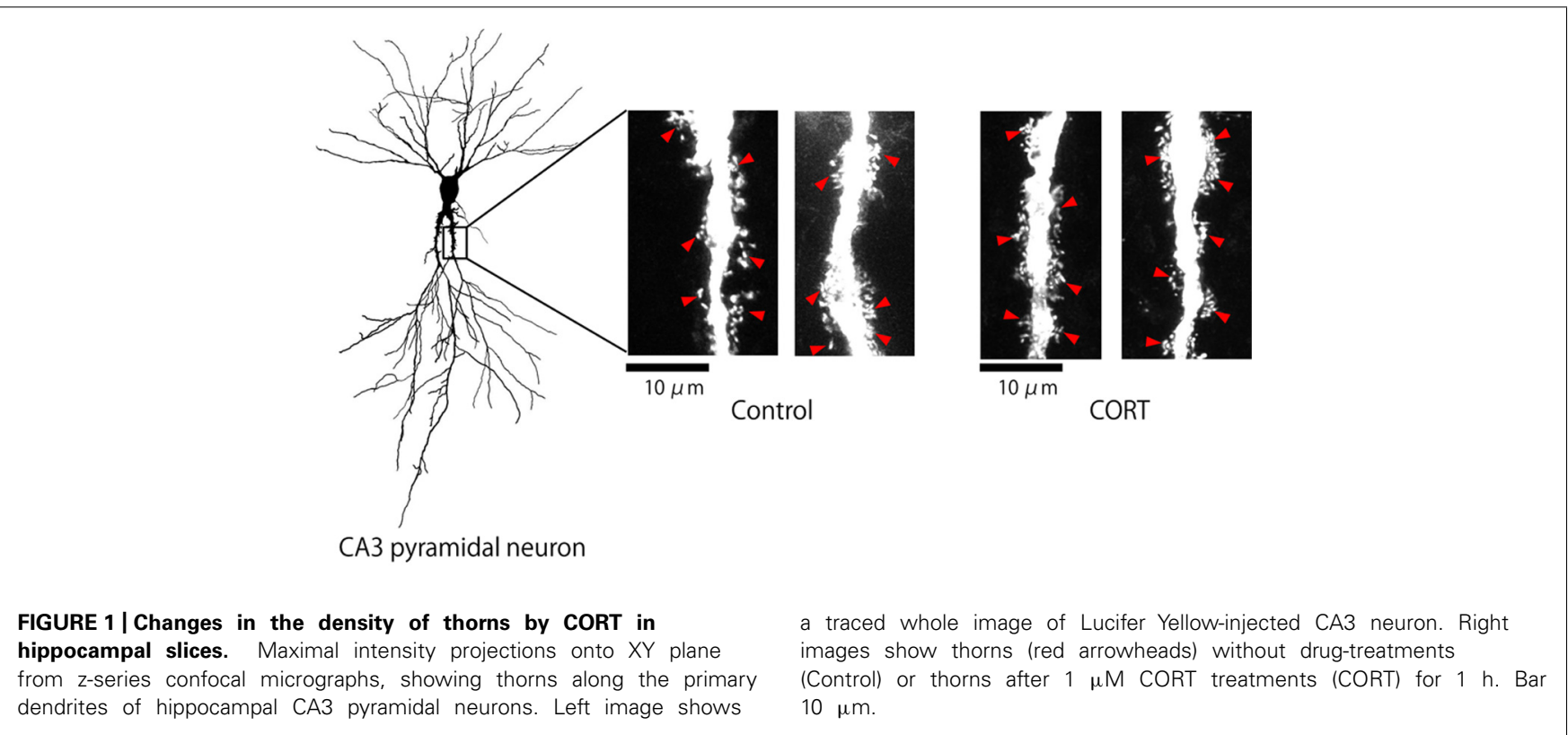


A

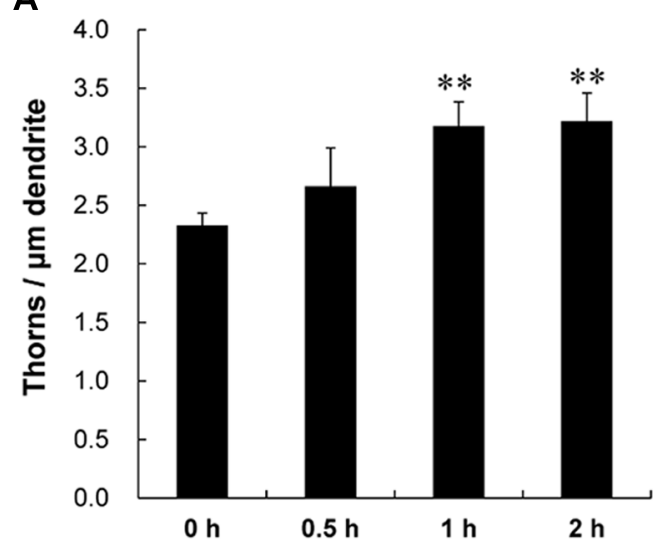

FIGURE 2 | Time dependency and dose dependency of CORT effects on the thorn density of CA3 neurons. Thorns were analyzed along the primary and secondary dendrites of pyramidal neurons in the stratum lucidum of CA3 neurons. (A) The time dependency of CORT effects on the thorn density in CA3 neurons, after $0.5 \mathrm{~h}$ treatment $(0.5 \mathrm{~h}), 1 \mathrm{~h}$ treatment $(1 \mathrm{~h})$, and $2 \mathrm{~h}$ treatment ( $2 \mathrm{~h}$ ) in ACSF with $1 \mu \mathrm{M}$ CORT. As a control, no treatment with CORT $(0 \mathrm{~h})$ is shown. (B) Dose dependency of CORT treatments on the thorn density. A $1 \mathrm{~h}$ treatment in ACSF without CORT $(0 \mathrm{nM})$, with $10 \mathrm{nM}$ CORT

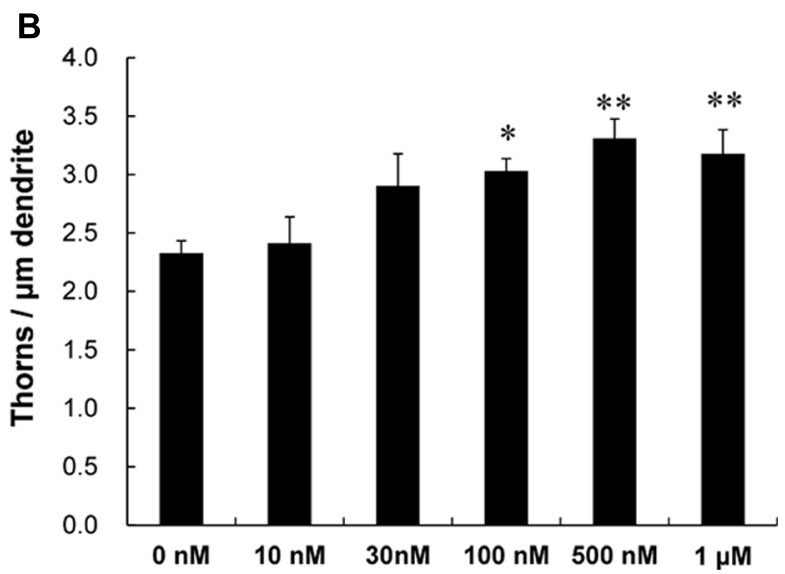

(10 nM), with $30 \mathrm{nM}$ CORT (30 nM), with $100 \mathrm{nM}$ CORT (100 nM), with $500 \mathrm{nM}$ CORT $(500 \mathrm{nM})$, and with $1 \mu \mathrm{M}$ CORT $(1 \mu \mathrm{M})$. Vertical axis is the average number of thorns per $1 \mu \mathrm{m}$ of dendrite. Results are reported as mean \pm SEM. The significance of CORT or drug effect was examined using the Tukey-Kramer post hoc multiple comparisons test when one way ANOVA tests yielded $P<0.05$. The significance yielded ${ }^{*} P<0.01{ }^{*} P<0.05$,

${ }^{*} P<0.01$ to $0 \mathrm{~h}$ and $0 \mathrm{nM}$. For each drug treatment, we investigated 3 rats, 6 slices, 12 neurons, 12 dendrites, and 1400-1800 thorns.

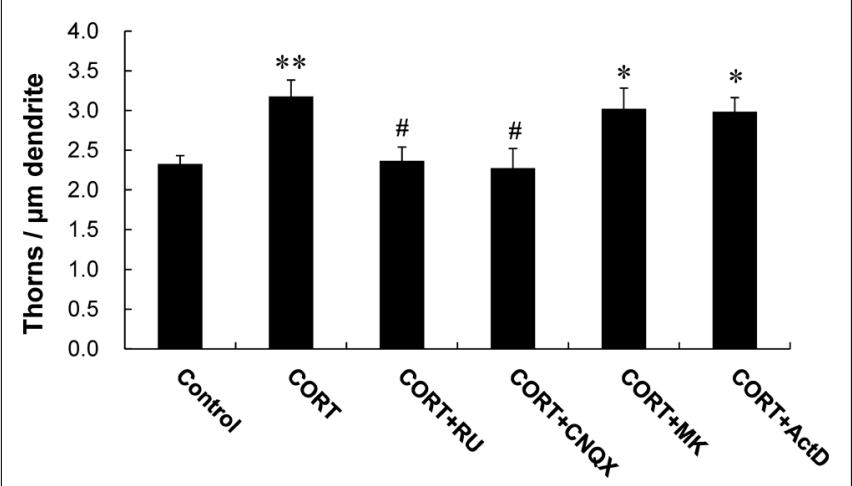

FIGURE 3 | Effects of blockers of receptors on CORT-induced changes in the thorn density. A $1 \mathrm{~h}$ treatment in ACSF without drugs (Control), with $1 \mu \mathrm{M}$ CORT (CORT), with 1 and $10 \mu \mathrm{M}$ RU486 (CORT + RU), with $1 \mu \mathrm{M}$ CORT and $20 \mu \mathrm{M}$ CNQX (CORT + CNOX), and with $1 \mu \mathrm{M}$ CORT and $50 \mu \mathrm{M}$ MK-801 (CORT + MK). Vertical axis is the average number of thorns per $1 \mu \mathrm{m}$ of dendrite. Results are reported as mean \pm SEM. The significance of CORT or drug effect was examined using the Tukey-Kramer post hoc multiple comparisons test when one way ANOVA tests yielded $P<0.05$. ${ }^{*} P<0.05$, ${ }^{*} P<0.01$ vs. Control. ${ }^{*} P<0.05$ vs. CORT. For each drug treatment, we investigated 3 rats, 6 slices, 12 neurons, 12 dendrites, and 1400-1800 thorns.

\section{Blocking of glutamate receptors abolished CORT-induced thorn-genesis}

We investigated the importance of $\mathrm{Ca}^{2+}$ homeostasis within thorns on CORT effects. Because the $\mathrm{Ca}^{2+}$ level may be maintained with spontaneous fluctuation of opening/closing via ionotropic glutamate receptors in thorns, we examined thorn-genesis in the presence of inhibitors of these receptors. 6-cyano-7-nitroquinoxaline-2,3-dione (CNQX), an inhibitor of $\alpha$-amino-3-hydroxy-5-methyl-4-isoxazolepropionate (AMPA) receptor, significantly suppressed the effect of CORT on the thorn density to 2.3 thorns/ $\mu \mathrm{m}$ (Figure 3). MK-801, an NMDA receptor blocker, did not abolish the CORT effect.

In additional experiments, Actinomycin D (ActD), an mRNA synthesis inhibitor, did not significantly suppress the CORTinduced increase in the density of thorns (Figure 3).

\section{Ultrastructural analysis for synaptic, extranuclear and nuclear localization of $\mathbf{G R}$}

To explain the site of rapid thorn-genesis by the activation of GR, a clarification of the subcellular localization (particularly the synaptic or extranuclear localization) of GR in CA3 pyramidal cells is essential. The synaptic, extranuclear and nuclear localization of GR was clarified via ultrastructural investigations using GR IgG (1/3000). An immunoelectron microscopic analysis using post-embedded immunogold was performed to determine the localization of GR-immunoreactivity in the hippocampal CA3 pyramidal cells. GR was localized not only in the nuclei but also in both the axon terminals and thorns of pyramidal cells (Figure 5A). At postsynapses, gold particles were distributed within the cytoplasm of the thorn head. Significant labeling along dendrites was also observed (Figure 5B). For a search of immunogold-labeled GR proteins, multiple labeling (three or more) of immunogold in the pre- and post-synaptic compartments was confirmed in at least 100 images. Each image contained several synapses among which at least one synapse expressed GR particles. We also observed some synapses in one image that did not express GR particles. Consequently, we observed approximately 10-20\% of synapses that expressed GR particles. Preadsorption of the antibody with GR antigen $(30 \mu \mathrm{g} / \mathrm{ml})$ resulted in the disappearance of immunoreactivity.

The antibody used for the current experiments were shown to have specific binding to GR in the hippocampus with Western 


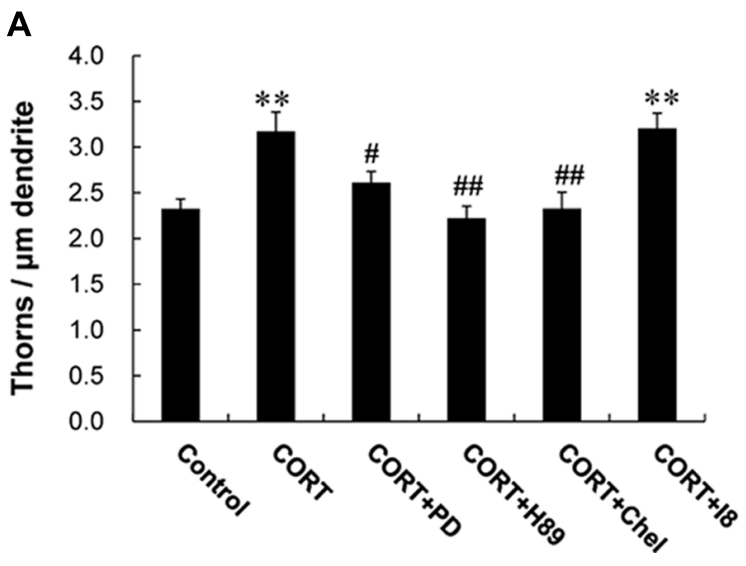

B

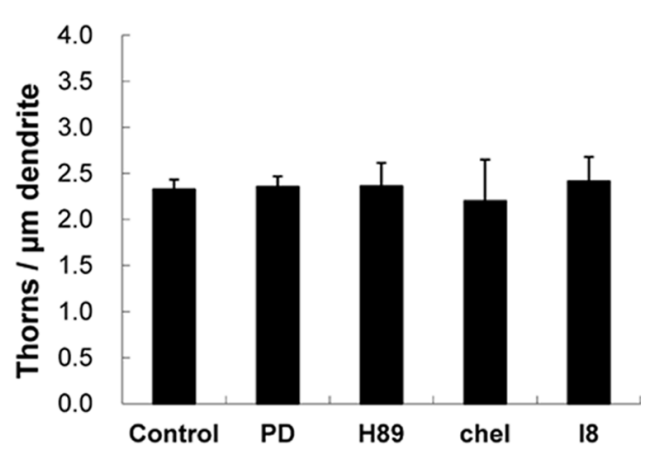

FIGURE 4 | Suppression effects by kinase inhibitors on CORT-induced changes in the density of thorns. (A) A $1 \mathrm{~h}$ treatment in ACSF without drugs (Control), with $1 \mu \mathrm{M}$ CORT (CORT), with $1 \mu \mathrm{M}$ CORT and $20 \mu \mathrm{M}$ PD98059 (MAPK inhibitor; CORT + PD), with $1 \mu \mathrm{M}$ CORT and $10 \mu \mathrm{M} \mathrm{H}-89$ (PKA inhibitor; CORT + H89), with $1 \mu \mathrm{M}$ CORT and $10 \mu \mathrm{M}$ chelerythrine (PKC inhibitor; CORT + Chel), and with $1 \mu \mathrm{M}$ CORT and $10 \mu \mathrm{M}$ GSK-3 $\beta$ Inhibitor VIII (CORT + 18). (B) No effect of kinase inhibitors alone on the density of thorns

\begin{abstract}
in CA3 neurons. Abbreviations are the same as (A). Vertical axis is the average number of thorns per $1 \mu \mathrm{m}$ of dendrite. Results are reported as mean \pm SEM. The significance of CORT or drug effect was examined using the Tukey-Kramer post hoc multiple comparisons test when one way ANOVA tests yielded $P<0.05$. ${ }^{* *} P<0.01$ vs. Control. ${ }^{\#} P<0.05$, ${ }^{\# \#} P<0.01$ vs. CORT. For each drug treatment, we investigated 3 rats, 6 slices, 12 neurons, 12 dendrites, and 1600-1800 thorns.
\end{abstract}
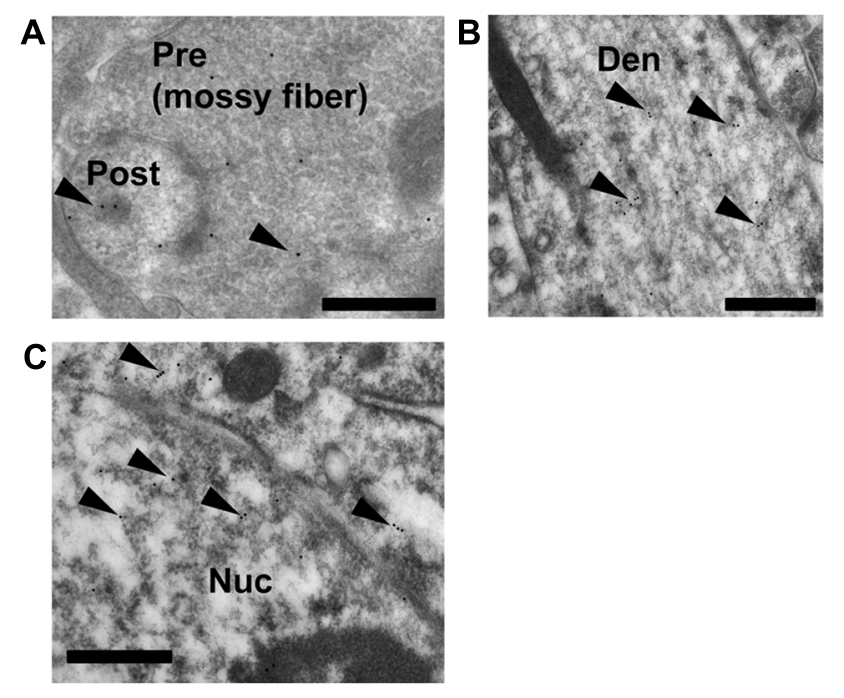

FIGURE 5 | Immunoelectron microscopic analysis of the distribution of GR within the mossy fiber synapses, dendrites in stratum lucidum and nuclei of pyramidal cells in CA3 region. Gold particles (arrowheads), specifically indicating the presence of GR, were localized in the pre- and postsynaptic regions (A). In dendrites, gold particles were often found in the cytoplasmic space (B). Gold particles were also localized in the nuclei (C). A search for immuno-gold labeled GR proteins was performed at least 30 synapses at CA3 region from more than 100 independent images. A 1:3000 dilution of IgG was used to prevent non-specific labeling. Pre, presynaptic region; Post, post synaptic region; Den, dendrite; Nuc, nucleus. Scale bar, $500 \mathrm{~nm}$.

blotting (Komatsuzaki etal., 2005; Ooishi et al., 2012b). As a negative control of GR specificity, no GR immunoreactivity was observed in the magnocellular division of the paraventricular nucleus (Morimoto et al., 1996).

\section{DISCUSSION}

The current study demonstrated GR- and kinase-dependent mechanisms of rapid CORT-induced thorn-genesis in CA3 pyramidal neurons of the adult male rat hippocampus. An extremely concentrated distribution of thorny excrescences, as compared with sparse distribution of spines located in other regions, such as CA1, prevented detailed analysis of thorny excrescences by previous studies using Golgi staining methods (Gould et al., 1990; Woolley etal., 1990a). We were able to analyze the number of thorns by the high-resolution image analysis of Lucifer Yellow-injected neurons, using deconvolution, and digital threedimensional analysis. Mossy fiber terminals originating from granule cells in DG provide excitatory inputs to CA3 neurons via thorny excrescences in the stratum lucidum (Amaral and Dent, 1981; Chicurel and Harris, 1992). Our data imply that CORT may rapidly enhance the excitatory input to CA3 from DG by increasing the density of thorns.

\section{STEROID LEVELS IN “ACUTE” SLICES USED FOR THORN EXPERIMENTS}

Following exposure to stress in rat, a high-dose of CORT (about $1 \mu \mathrm{M})$ is secreted by the adrenal cortex and readily penetrates into the brain from the blood circulation. The steroid levels in slices used for thorn analysis must be known. From our earlier study (Komatsuzaki et al., 2012), the CORT concentration in the freshly isolated hippocampus was $400-1000 \mathrm{nM}$ as determined by mass-spectrometric analysis (Hojo et al., 2009, 2011; Ooishi et al., 2012a,b), because rats were under decapitation stress which caused penetration of elevated plasma CORT (1-2 $\mu \mathrm{M})$ into the hippocampus (Pardridge and Mietus, 1979). Note that 1-2 $\mu \mathrm{M}$ CORT is more than the upper limit capacity $(400-600 \mathrm{nM})$ of CORT binding globulin (Breuner and Orchinik, 2002). However, the control "acute" slices, used for the thorn analysis, have very low CORT level of approximaterly $2 \mathrm{nM}$ by recovery incubation of "fresh" slices (with high CORT concentration of 400-1000 nM) 
in steroid-free ACSF for $2 \mathrm{~h}$, due to leakage of CORT from slices to ACSF (Hojo et al., 2011; Ooishi et al., 2012a,b). This CORT leakage always occurs in hippocampal slices prepared at different time (morning, afternoon, or evening), therefore control acute slices should have always low CORT. From these reasons, the enhanced thorn-genesis occurred upon increase in CORT level from approximately $2 \mathrm{nM}$ (control) to $100-1000 \mathrm{nM}$ by CORT application.

\section{CONTRIBUTION OF SYNAPTIC/EXTRANUCLEAR GR TO THE RAPID MODULATION}

Since the effect of CORT had been blocked by RU486, we confirmed that CORT-effect was directly mediated by GR. The expression of GR in the CA3 region was demonstrated by immunoelectron microscopy, although the GR expression in CA3 was a little bit weak in immunohistochemistry and in situ hybridization, in comparison with CA1 (Morimoto et al., 1996). We observed GR localized within the postsynaptic structures via postembedding immunogold staining (Figure 5). The current CORT treatment may rapidly activate the synaptic GR. GR was often located in the cytoplasm of thorns (Komatsuzaki et al., 2005; Ooishi et al., 2012b). GR was observed also in dendrites and nuclei (Komatsuzaki et al., 2005; Ooishi et al., 2012b). Our earlier study shows that GR also expressed in purified PSD fraction by Western blot analysis (Komatsuzaki et al., 2005; Ooishi et al., 2012b). These results suggest that the rapid modulation of thorns by CORT may be mediated by postsynaptic or extranuclear GR. The involvement of GR in the CORT effect was also supported by GR antagonist RU486 which blocked CORT-induced thorn-genesis (Figure 3). In addition, GR agonist dexamethasone induces rapid spinogenesis in hippocampal CA1 neurons within $1 \mathrm{~h}$ (Komatsuzaki et al., 2005). Membrane GR-induced rapid PKA activation ( $\sim 1 \mathrm{~h})$ has been demonstrated for inhibitory avoidance behavior via rat basolateral amygdala (Roozendaal et al., 2002), suggesting that synaptic GR may activate kinases.
Since in our "acute" slice CORT level is around $2 \mathrm{nM}$, all mineral corticoid receptor (MR) may be occupied due to high affinity $(\mathrm{Kd} \sim 0.5 \mathrm{nM})$ to aldosterone or CORT. Therefore the effect of CORT on thorns is mainly mediated by GR but not by MR, in the presence of $1 \mu \mathrm{M}$ CORT. The $1 \mathrm{~h}$ responses may be too rapid for nuclear GR actions which often need more than $5 \mathrm{~h}$ due to genetic processes. As an another possibility, these rapid actions may include rapid genomic actions via nuclear GR, which are suggested as a reduction of dendritic length (1-4 h; Alfarez et al., 2009) or an impairment of enhancement of voltage-dependent $\mathrm{Ca}^{2+}$ currents in mutated GR (1-4 h; Karst et al., 2000), in hippocampal neurons. However, since ActD did not suppress the CORT-induced thorn-increase (Figure 3), rapid transcriptional process probably does not participate in the thorn-genesis. A significant suppression of thorn changes by application of kinase inhibitors (Figure 4) may put more weight on GR-kinase pathway rather than DNA binding of GR.

Since RU486 suppresses not only GR but also progesterone receptor (PR) in Figure 3, progesterone (PROG) effect should be considered. The treatment of slices with $10 \mathrm{nM}$ PROG for $1 \mathrm{~h}$ did not significantly increase the thorn density within experimental error (data not shown), excluding the involvement of PROG and $\mathrm{PR}$ in the observed thorn-genesis.

\section{CORT-INDUCED THORN-GENESIS VIA KINASE NETWORKS AND THEIR DOWNSTREAM}

There is increasing evidence implying that CORT is capable of driving rapid signaling (around $1 \mathrm{~h}$ ), independent of slow transcriptional signaling (model illustration in Figure 6). Rapid MAPK activation $(\sim 2 \mathrm{~h})$ via GR has been demonstrated in the mice hippocampus or pituitary-derived cell-lines AtT20 (Revest et al., 2005). The expression of Raf1, Ras, p-MAPK is elevated rapidly upon application of $10 \mathrm{nM}$ CORT. Fear conditioning of mice is dependent on GR-MAPK pathway. Rapid PKA activation (phosphorylation of PKA; $\sim 1 \mathrm{~h}$ ) via membrane located GR has

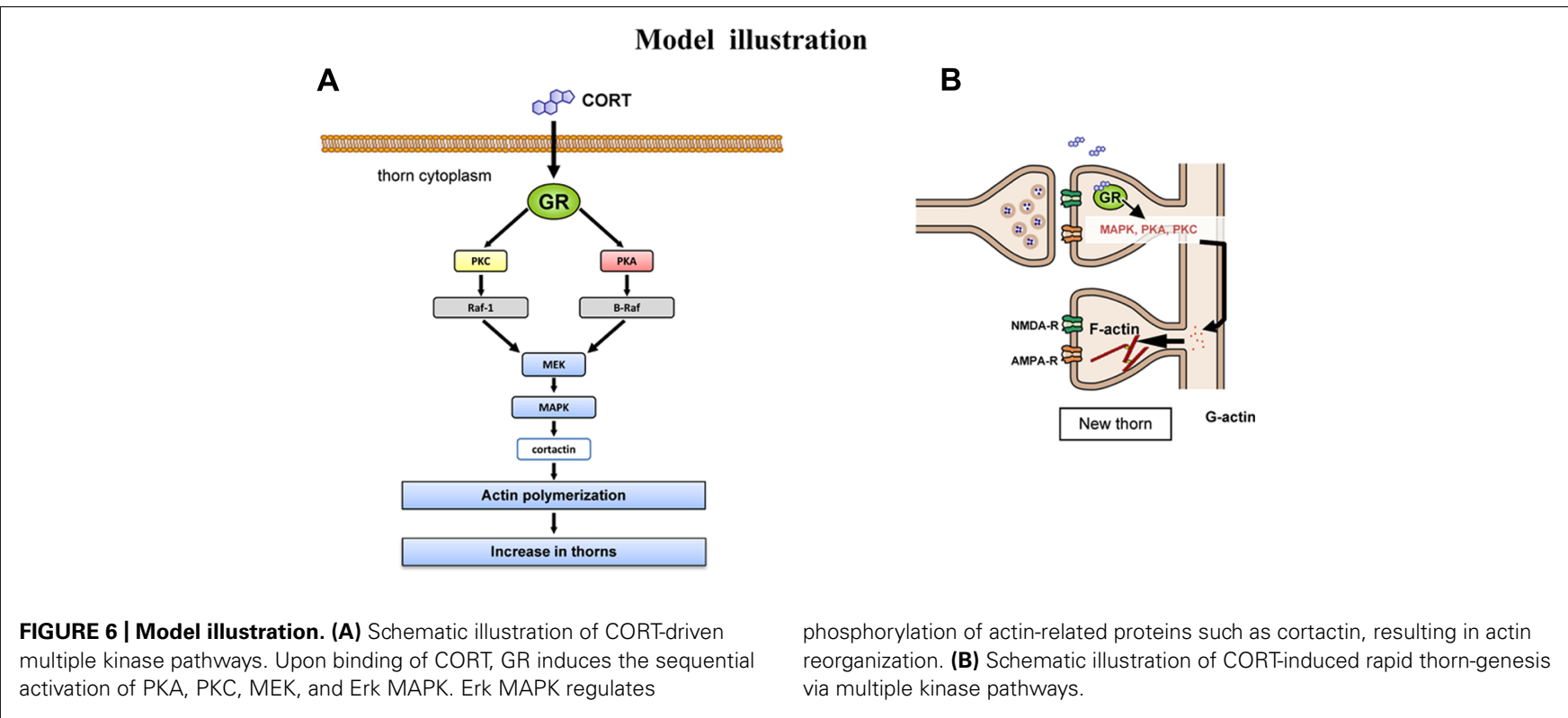


been demonstrated in rat basolateral amygdala (Roozendaal et al., 2002).

To consider the molecular mechanisms of kinase signaling in the modulation of CA3 thorn, we temporarily use the model of CA1 region. In this model, MAPK cascade is coupled with PKA and $\mathrm{PKC}$ via $\mathrm{PKC} \rightarrow \mathrm{Rafl} \rightarrow \mathrm{MAPK}, \mathrm{PKA} \rightarrow \mathrm{B}-\mathrm{Raf} \rightarrow \mathrm{MAPK}$ in synaptic modulation (Adams et al., 2000; Komatsuzaki et al., 2012). Taking the knowledge into account, MAPK may be a key kinase responsible for modulation of thorns. The target of Erk MAPK in thorn reorganization is cortactin, since Erk MAPK is known to phosphorylate cortactin, a structural protein associated to actin (MacQueen et al., 2003). Cortactin interacts with both F-actin and actin-related protein (Arp) 2/3 complex as well as scaffold protein Shank in the PSD at the SH3 domain (Weed et al., 1998; Daly, 2004), resulting in promotion of actin fiber remodeling within spines or thorns.

It is thus probable that CORT exerts its effect on thorns via cortactin-actin pathway. Cortactin has multiple phosphorylation sites which are activated by MAPK (Campbell et al., 1999). Phosphorylation of cortactin may promote assembly of actin cytoskeletal matrices, resulting in thorn formation or modulation of thorn morphology (Hering and Sheng, 2003). These sites are putative phosphorylation sites also for other serine/threonine kinase (PKA or PKC) that are activated by CORT.

In the case of in vivo hippocampus, the similar rapid CORTinduced modulation of thorns might occur. In response to acute severe stress, elevation of plasma CORT (to 1-2 $\mu \mathrm{M}$ ) occurs, resulting in elevation of hippocampal CORT to $0.5-1 \mu \mathrm{M}$, due to penetration of CORT into hippocampus after crossing the Blood Brain Barrier (Higo etal., 2011). This increase of CORT level should affect thorn-genesis in vivo.

The abolishment of the CORT-induced increase in the density of thorns by CNQX (Figure 3) suggests the correlation of the CORT signaling pathway with AMPA receptors. Maintenance of the suitable basal $\mathrm{Ca}^{2+}$ level may be important for action of PKC or MAPK on thorn-genesis. CNQX may decrease the basal $\mathrm{Ca}^{2+}$ level which is spontaneously formed by ion exchange systems consisting of AMPA receptors plus voltage activated calcium channels. This explanation is deduced from previous study which has shown that the $\mathrm{Ca}^{2+}$ influx within thorny excrescences has occurred via AMPA receptor-dependent voltage activated calcium channels during subthreshold activation of CA3 neurons, while NMDA receptors-mediated calcium influx within CA3 thorny excrescences is smaller than that in CA1 spines upon subthreshold activation (Monaghan et al., 1983; Baude et al., 1995; Fritschy et al., 1999; Reid et al., 2001; Reid, 2002).

\section{OTHER EXAMPLES OF KINASE-DEPENDENT THORN-GENESIS OR SPINOGENESIS}

The activation of synaptic androgen receptor AR by testosterone or dihydrotestosterone induces a rapid increase of thorns of thorny excrescences in CA3 pyramidal neurons of adult rat hippocampal "acute" slices within $2 \mathrm{~h}$. The rapid synaptic action of androgen is also mediated by activation of many kinases. This thorn-genesis induced by androgen is mediated by Erk MAPK, p38 MAPK, PKC, CaMKII, but not by PKA and PI3K (Hatanaka et al., 2009). The rapid spinogenesis by estradiol in CA3 and CA1 pyramidal neurons of hippocampal "acute" slices is mediated by synaptic $\mathrm{ER} \alpha \rightarrow$ Erk MAPK pathway (Tsurugizawa et al., 2005; Mukai et al., 2007).

Concerning CA3 rapid stress effects, the administration of corticotropin releasing hormone $(\mathrm{CRH})$, rapidly (within $0.5 \mathrm{~h}$ ) induces loss of CA3 dendritic spines (different from thorns) in stratum radiatum, using Yellow Fluorescence Protein (YFP)expressing hippocampal neurons (Chen et al., 2008).

One of the physiological significance of GR-induced increase in thorns may be that upon acute stress (for example, stress at examination or oral interview) neurons may be activated and new thorns may appear, resulting in synaptic remodeling. This is very different from chronic stress-induced depression of neural activities via nuclear GR-induced genetic transcription processes.

\section{REFERENCES}

Adams, J. P., Roberson, E. D., English, J. D., Selcher, J. C., and Sweatt, J. D. (2000). MAPK regulation of gene expression in the central nervous system. Acta Neurobiol. Exp. (Wars.) 60, 377-394.

Alfarez, D. N., De Simoni, A., Velzing, E. H., Bracey, E., Joels, M., Edwards, F. A., et al. (2009). Corticosterone reduces dendritic complexity in developing hippocampal CA1 neurons. Hippocampus 19, 828-836. doi: 10.1002/hipo.20566

Alonso, M., Medina, J. H., and Pozzo-Miller, L. (2004). ERK1/2 activation is necessary for BDNF to increase dendritic spine density in hippocampal CA1 pyramidal neurons. Learn. Mem. 11, 172-178. doi: 10.1101/lm.67804

Amaral, D. G., and Dent, J. A. (1981). Development of the mossy fibers of the dentate gyrus: I. A light and electron microscopic study of the mossy fibers and their expansions. J. Comp. Neurol. 195, 51-86. doi: 10.1002/cne.901950106

Baude, A., Nusser, Z., Molnar, E., Mcilhinney, R. A., and Somogyi, P. (1995). Highresolution immunogold localization of AMPA type glutamate receptor subunits at synaptic and non-synaptic sites in rat hippocampus. Neuroscience 69, 1031-1055. doi: 10.1016/0306-4522(95)00350-R

Bhat, R., Xue, Y., Berg, S., Hellberg, S., Ormo, M., Nilsson, Y., et al. (2003). Structural insights and biological effects of glycogen synthase kinase 3-specific inhibitor AR-A014418. J. Biol. Chem. 278, 45937-45945. doi: 10.1074/jbc.M306268200

Birnbaum, S. G., Yuan, P. X., Wang, M., Vijayraghavan, S., Bloom, A. K., Davis, D. J., et al. (2004). Protein kinase $C$ overactivity impairs prefrontal cortical regulation of working memory. Science 306, 882-884. doi: 10.1126/science.1100021

Breuner, C. W., and Orchinik, M. (2002). Plasma binding proteins as mediators of corticosteroid action in vertebrates. J. Endocrinol. 175, 99-112. doi: 10.1677/joe.0.1750099

Campbell, D. H., Sutherland, R. L., and Daly, R. J. (1999). Signaling pathways and structural domains required for phosphorylation of EMS1/cortactin. Cancer Res. 59, 5376-5385.

Chen, Y., Dube, C. M., Rice, C. J., and Baram, T. Z. (2008). Rapid loss of dendritic spines after stress involves derangement of spine dynamics by corticotropinreleasing hormone. J. Neurosci. 28, 2903-2911. doi: 10.1523/JNEUROSCI.022508.2008

Chen, Q., Zhou, Z., Zhang, L., Wang, Y., Zhang, Y. W., Zhong, M., etal. (2012). Tau protein is involved in morphological plasticity in hippocampal neurons in response to BDNF. Neurochem. Int. 60, 233-242. doi: 10.1016/j.neuint.2011.12.013

Chicurel, M. E., and Harris, K. M. (1992). Three-dimensional analysis of the structure and composition of CA3 branched dendritic spines and their synaptic relationships with mossy fiber boutons in the rat hippocampus. J. Comp. Neurol. 325, 169-182. doi: 10.1002/cne.903250204

Chijiwa, T., Mishima, A., Hagiwara, M., Sano, M., Hayashi, K., Inoue, T., et al. (1990). Inhibition of forskolin-induced neurite outgrowth and protein phosphorylation by a newly synthesized selective inhibitor of cyclic AMP-dependent protein kinase, $\mathrm{N}$-[2-(p-bromocinnamylamino)ethyl]-5-isoquinolinesulfonamide (H-89), of PC12D pheochromocytoma cells. J. Biol. Chem. 265, 5267-5272.

Daly, R. J. (2004). Cortactin signalling and dynamic actin networks. Biochem. J. 382, 13-25. doi: 10.1042/BJ20040737

Diamond, D. M., Bennett, M. C., Fleshner, M., and Rose, G. M. (1992). Inverted-U relationship between the level of peripheral corticosterone and the magnitude 
of hippocampal primed burst potentiation. Hippocampus 2, 421-430. doi: 10.1002/hipo.450020409

Duan, H., Wearne, S. L., Morrison, J. H., and Hof, P. R. (2002). Quantitative analysis of the dendritic morphology of corticocortical projection neurons in the macaque monkey association cortex. Neuroscience 114, 349-359. doi: 10.1016/S0306-4522(02)00305-6

Dudley, D. T., Pang, L., Decker, S. J., Bridges, A. J., and Saltiel, A. R. (1995). A synthetic inhibitor of the mitogen-activated protein kinase cascade. Proc. Natl. Acad. Sci. U.S.A. 92, 7686-7689. doi: 10.1073/pnas.92.17.7686

Fritschy, J. M., Meskenaite, V., Weinmann, O., Honer, M., Benke, D., and Mohler, H. (1999). GABAB-receptor splice variants GB1a and GB1b in rat brain: developmental regulation, cellular distribution and extrasynaptic localization. Eur. J. Neurosci. 11, 761-768. doi: 10.1046/j.1460-9568.1999.00481.x

Gould, E., Woolley, C. S., Frankfurt, M., and Mcewen, B. S. (1990). Gonadal steroids regulate dendritic spine density in hippocampal pyramidal cells in adulthood. $J$. Neurosci. 10, 1286-1291.

Hammond, R. S., Lin, L., Sidorov, M. S., Wikenheiser, A. M., and Hoffman, D. A. (2008). Protein kinase a mediates activity-dependent Kv4.2 channel trafficking. J. Neurosci. 28, 7513-7519. doi: 10.1523/JNEUROSCI.1951-08.2008

Hatanaka, Y., Mukai, H., Mitsuhashi, K., Hojo, Y., Murakami, G., Komatsuzaki, Y., et al. (2009). Androgen rapidly increases dendritic thorns of CA3 neurons in male rat hippocampus. Biochem. Biophys. Res. Commun. 381, 728-732. doi: 10.1016/j.bbrc.2009.02.130

Herbert, J. M., Augereau, J. M., Gleye, J., and Maffrand, J. P. (1990). Chelerythrine is a potent and specific inhibitor of protein kinase C. Biochem. Biophys. Res. Commun. 172, 993-999. doi: 10.1016/0006-291X(90)91544-3

Hering, H., and Sheng, M. (2003). Activity-dependent redistribution and essential role of cortactin in dendritic spine morphogenesis. J. Neurosci. 23, 11759-11769.

Higo, S., Hojo, Y., Ishii, H., Komatsuzaki, Y., Ooishi, Y., Murakami, G., et al. (2011). Endogenous synthesis of corticosteroids in the hippocampus. PLoS ONE 6:e21631. doi: 10.1371/journal.pone.0021631

Hojo, Y., Hattori, T. A., Enami, T., Furukawa, A., Suzuki, K., Ishii, H. T., et al (2004). Adult male rat hippocampus synthesizes estradiol from pregnenolone by cytochromes P45017alpha and P450 aromatase localized in neurons. Proc. Natl. Acad. Sci. U.S.A. 101, 865-870. doi: 10.1073/pnas.2630225100

Hojo, Y., Higo, S., Ishii, H., Ooishi, Y., Mukai, H., Murakami, G., et al. (2009). Comparison between hippocampus-synthesized and circulation-derived sex steroids in the hippocampus. Endocrinology 150, 5106-5112. doi: 10.1210/en.2009-0305

Hojo, Y., Higo, S., Kawato, S., Hatanaka, Y., Ooishi, Y., Murakami, G., et al. (2011). Hippocampal synthesis of sex steroids and corticosteroids: essential for modulation of synaptic plasticity. Front. Endocrinol. 2:43. doi: 10.3389/fendo.2011.00043

Karst, H., Karten, Y. J., Reichardt, H. M., De Kloet, E. R., Schutz, G., and Joels, M. (2000). Corticosteroid actions in hippocampus require DNA binding of glucocorticoid receptor homodimers. Nat. Neurosci. 3, 977-978. doi: $10.1038 / 79910$

Komatsuzaki, Y., Hatanaka, Y., Murakami, G., Mukai, H., Hojo, Y., Saito, M., et al. (2012). Corticosterone induces rapid spinogenesis via synaptic glucocorticoid receptors and kinase networks in hippocampus. PLoS ONE 7:e34124. doi: 10.1371/journal.pone.0034124

Komatsuzaki, Y., Murakami, G., Tsurugizawa, T., Mukai, H., Tanabe, N., Mitsuhashi, K., et al. (2005). Rapid spinogenesis of pyramidal neurons induced by activation of glucocorticoid receptors in adult male rat hippocampus. Biochem. Biophys. Res. Commun. 335, 1002-1007. doi: 10.1016/j.bbrc.2005.07.173

Lupien, S. J., and McEwen, B. S. (1997). The acute effects of corticosteroids on cognition: integration of animal and human model studies. Brain Res. Brain Res. Rev. 24, 1-27. doi: 10.1016/S0165-0173(97)00004-0

MacQueen, G. M., Campbell, S., Mcewen, B. S., Macdonald, K., Amano, S., Joffe, R. T., et al. (2003). Course of illness, hippocampal function, and hippocampal volume in major depression. Proc. Natl. Acad. Sci. U.S.A. 100, 1387-1392. doi: 10.1073/pnas.0337481100

McNaughton, B. L., and Morris, R. G. M. (1987). Hippocampal synaptic enhancement and information-storage within a distributed memory system. Trends Neurosci. 10, 408-415. doi: 10.1016/0166-2236(87)90011-7

Monaghan, D. T., Holets, V. R., Toy, D. W., and Cotman, C. W. (1983). Anatomical distributions of four pharmacologically distinct $3 \mathrm{H}$-L-glutamate binding sites. Nature 306, 176-179. doi: 10.1038/306176a0

Morimoto, M., Morita, N., Ozawa, H., Yokoyama, K., and Kawata, M. (1996). Distribution of glucocorticoid receptor immunoreactivity and mRNA in the rat brain: an immunohistochemical and in situ hybridization study. Neurosci. Res 26, 235-269. doi: 10.1016/S0168-0102(96)01105-4

Morris, R. G., Garrud, P., Rawlins, J. N., and O'Keefe, J. (1982). Place navigation impaired in rats with hippocampal lesions. Nature 297, 681-683. doi: $10.1038 / 297681 \mathrm{a0}$

Mukai, H., Hatanaka, Y., Mitsuhashi, K., Hojo, Y., Komatsuzaki, Y., Sato, R., etal. (2011). Automated analysis of spines from confocal laser microscopy images: application to the discrimination of androgen and estrogen effects on spinogenesis. Cereb. Cortex 21, 2704-2711. doi: 10.1093/cercor/ bhr059

Mukai, H., Tsurugizawa, T., Murakami, G., Kominami, S., Ishii, H., Ogiue-Ikeda, M., et al. (2007). Rapid modulation of long-term depression and spinogenesis via synaptic estrogen receptors in hippocampal principal neurons. J. Neurochem. 100, 950-967. doi: 10.1111/j.1471-4159.2006.04264.x

Ooishi, Y., Kawato, S., Hojo, Y., Hatanaka, Y., Higo, S., Mukai, H., et al. (2012a). Modulation of synaptic plasticity in the hippocampus by hippocampusderived estrogen and androgen. J. Steroid Biochem. Mol. Biol. 131, 37-51. doi: 10.1016/j.jsbmb.2011.10.004

Ooishi, Y., Mukai, H., Hojo, Y., Murakami, G., Hasegawa, Y., Shindo, T., et al. (2012b). Estradiol rapidly rescues synaptic transmission from corticosteroneinduced suppression via synaptic/extranuclear steroid receptors in the hippocampus. Cereb. Cortex 22, 926-936. doi: 10.1093/cercor/bhr164

Pardridge, W. M., and Mietus, L. J. (1979). Transport of steroid hormones through the rat blood-brain barrier. Primary role of albumin-bound hormone. J. Clin. Invest. 64, 145-154. doi: 10.1172/JCI109433

Reagan, L. P., and McEwen, B. S. (1997). Controversies surrounding glucocorticoidmediated cell death in the hippocampus. J. Chem. Neuroanat. 13, 149-167. doi: 10.1016/S0891-0618(97)00031-8

Reid, C. A. (2002). The role of dendritic spines: comparing the complex with the simple. Eur. J. Pharmacol. 447, 173-176. doi: 10.1016/S0014-2999(02) 01841-1

Reid, C. A., Fabian-Fine, R., and Fine, A. (2001). Postsynaptic calcium transients evoked by activation of individual hippocampal mossy fiber synapses. J. Neurosci. 21, 2206-2214.

Revest, J. M., Di Blasi, F., Kitchener, P., Rouge-Pont, F., Desmedt, A., Turiault, M., et al. (2005). The MAPK pathway and Egr-1 mediate stress-related behavioral effects of glucocorticoids. Nat. Neurosci. 8, 664-672. doi: 10.1038/nn1441

Roberson, E. D., English, J. D., Adams, J. P., Selcher, J. C., Kondratick, C., and Sweatt, J. D. (1999). The mitogen-activated protein kinase cascade couples PKA and $\mathrm{PKC}$ to cAMP response element binding protein phosphorylation in area CA1 of hippocampus. J. Neurosci. 19, 4337-4348.

Roozendaal, B., Quirarte, G. L., and Mcgaugh, J. L. (2002). Glucocorticoids interact with the basolateral amygdala beta-adrenoceptor - cAMP/cAMP/PKA system in influencing memory consolidation. Eur. J. Neurosci. 15, 553-560. doi: 10.1046/j.0953-816x.2001.01876.x

Sato, S., Osanai, H., Monma, T., Harada, T., Hirano, A., Saito, M., et al. (2004). Acute effect of corticosterone on $N$-methyl-D-aspartate receptor-mediated $\mathrm{Ca}^{2+}$ elevation in mouse hippocampal slices. Biochem. Biophys. Res. Commun. 321, 510-513. doi: 10.1016/j.bbrc.2004.06.168

Shibuya, K., Takata, N., Hojo, Y., Furukawa, A., Yasumatsu, N., Kimoto, T., et al. (2003). Hippocampal cytochrome P450s synthesize brain neurosteroids which are paracrine neuromodulators of synaptic signal transduction. Biochim. Biophys. Acta 1619, 301-316. doi: 10.1016/S0304-4165(02)00489-0

Sorrells, S. F., Caso, J. R., Munhoz, C. D., and Sapolsky, R. M. (2009). The stressed CNS: when glucocorticoids aggravate inflammation. Neuron 64, 33-39. doi: 10.1016/j.neuron.2009.09.032

Stewart, M. G., Davies, H. A., Sandi, C., Kraev, I. V., Rogachevsky, V. V., Peddie, C. J., et al. (2005). Stress suppresses and learning induces plasticity in CA3 of rat hippocampus: a three-dimensional ultrastructural study of thorny excrescences and their postsynaptic densities. Neuroscience 131, 43-54. doi: 10.1016/j.neuroscience.2004.10.031

Tsurugizawa, T., Mukai, H., Tanabe, N., Murakami, G., Hojo, Y., Kominami, S., et al. (2005). Estrogen induces rapid decrease in dendritic thorns of CA3 pyramidal neurons in adult male rat hippocampus. Biochem. Biophys. Res. Commun. 337, 1345-1352. doi: 10.1016/j.bbrc.2005.09.188

Watanabe, Y., Gould, E., and Mcewen, B. S. (1992). Stress induces atrophy of apical dendrites of hippocampal CA3 pyramidal neurons. Brain Res. 588, 341-345. doi: 10.1016/0006-8993(92)91597-8 
Weed, S. A., Du, Y., and Parsons, J. T. (1998). Translocation of cortactin to the cell periphery is mediated by the small GTPase Racl. J. Cell Sci. 111(Pt 16), 2433-2443.

Woolley, C. S., Gould, E., Frankfurt, M., and McEwen, B. S. (1990a). Naturally occurring fluctuation in dendritic spine density on adult hippocampal pyramidal neurons. J. Neurosci. 10, 4035-4039.

Woolley, C. S., Gould, E., and McEwen, B. S. (1990b). Exposure to excess glucocorticoids alters dendritic morphology of adult hippocampal pyramidal neurons. Brain Res. 531, 225-231. doi: 10.1016/0006-8993(90) 90778-A

Conflict of Interest Statement: The authors declare that the research was conducted in the absence of any commercial or financial relationships that could be construed as a potential conflict of interest.
Received: 27 June 2013; accepted: 11 November 2013; published online: 27 November 2013.

Citation: Yoshiya M, Komatsuzaki Y, Hojo Y, Ikeda M, Mukai H, Hatanaka Y, Murakami G, Kawata M, Kimoto T and Kawato S (2013) Corticosterone rapidly increases thorns of CA3 neurons via synaptic/extranuclear glucocorticoid receptor in rat hippocampus. Front. Neural Circuits 7:191. doi: 10.3389/fncir.2013.00191

This article was submitted to the journal Frontiers in Neural Circuits.

Copyright (c) 2013 Yoshiya, Komatsuzaki, Hojo, Ikeda, Mukai, Hatanaka, Murakami, Kawata, Kimoto and Kawato. This is an open-access article distributed under the terms of the Creative Commons Attribution License (CC BY). The use, distribution or reproduction in other forums is permitted, provided the original author(s) or licensor are credited and that the original publication in this journal is cited, in accordance with accepted academic practice. No use, distribution or reproduction is permitted which does not comply with these terms. 